\title{
Research on Image Acquisition Method Model Based on Implicit Association Test
}

\author{
Zhuen $\mathrm{Guo}^{1}$ and $\mathrm{Li} \mathrm{Lin}^{2 *}$ \\ ${ }^{1}$ Key Laboratory of Advanced Manufacturing Technology, Ministry of Education, Guizhou University, Guiyang, Guizhou, 550025, \\ China \\ ${ }^{2}$ School of Mechanical Engineering, Guizhou University, Guiyang, Guizhou, 550025, China
}

\begin{abstract}
In the process of the traditional quantitative method is easily interfered with by subjective and external environment, and cannot reflect the real emotion of users. The implicit measurement method can better reflect the cognitive of users and has good reliability in perceptual evaluation. In this paper, the implicit cognitive processing process in users' perceptual evaluation of products is quantitatively analyzed. The correlation between product image attribute values and implicit measurement data is obtained. Thus, an image extraction model based on implicit measurement data is obtained. The implicit association test is introduced into the image extraction process, and the relationship between the implicit association test data of users and the data of product image attribute values is analyzed. Taking UAV as the analysis prototype, the image extraction model is obtained. After verification and analysis, the image extraction results are consistent with the image attribute values.
\end{abstract}

\section{Introduction}

With to improve consumption level, people are not satisfied with the single products, and the ancillary value and attribute meaning of products have become the focus of people's attention [1]. Users have gradually become the driving force for product innovation, and their needs are called the focus of design [2]. As the only user knowledge, user perceptual image preference is important information for design [3]. For the cognitive-perceptual information of the group, designers usually use explicit measurement methods such as questionnaires, brainstorming, oral analysis, focus groups. [4]. This kind of method has the advantages of simplicity and ease of use, flexible implementation, and simple data analysis. But it also has many shortcomings: for the explicit behaviors displayed by users after their subjective consciousness, users are vulnerable to external interference, resulting in users reducing their true consciousness of expressing their hearts. If users want to express real consciousness, they will often be affected by the experience, knowledge level, etc., and their explicit behaviors cannot be controlled by real consciousness [5]. Therefore, the traditional explicit measurement method can only analyze the explicit behavior of users, cannot detect the real conscious cognitive process of users, and cannot reach a highreliability level [6]. For the complexity of the human cognitive process and the subjectivity of product image evaluation, it is necessary to set up an objective image evaluation model from the perspective of the human cognitive process.
To make up for the shortage of explicit measurement, researchers began to introduce implicit measurement methods. This originally used in basic research fields such as psychological and cognitive into user research to explore the real needs. To experiences and internal cognitive processing of users and improve the reliability of user research [7-8]. To develop implicit measurement technology provides a new direction for the research of the user's image awareness [9]. In psychology, the most common implicit measurement method to explore users' real attitudes is the implicit association test [10]. In this paper, implicit association test technology is introduced into image acquisition to make it conform to image extraction. The model between implicit association test data and image value is established to get objective product image data and avoid the subjectivity of traditional image cognitive extraction.

\section{Image Acquisition Method Model Based on Implicit Association Test}

According to the analysis of relevant theories and technologies, an image acquisition method model based on the implicit association is established, and the mapping between implicit association test data and image values is established, to reflect the real needs of users with objective data.

\subsection{Establishing Image Semantic Space}

Through to collect relevant sample images in relevant websites, books, and other relevant materials, through to 
screen sample images, get the sample images that can objectively and accurately reflect, and establish the sample image set $U=\left\{U_{1}, U_{2}, U_{3}, \cdots, U_{u}\right\}$. At the same time of collecting sample pictures, image adjectives related to sample pictures are collected. After the selection and clustering of adjectives, semantic set $S=$ $\left\{S_{1}, S_{2}, S_{3}, \cdots, S_{s}\right\}$ is established and similar image semantic phrases are established. Through the survey, we can get the average images value $V$ under each image meaning of the samples.

\subsection{Screening of Experimental Samples}

To reduce the influence of subjective on the values of samples under various images, image semantic space will be established and then classified again. The questionnaire was established again and designed with three semantic differences, conforming to the image, conforming to the relative image and neutral information. The samples were studied again under each image to get new survey data. Comparing the survey data with the classified data, screening out the mismatched sample information between the two, getting further screened samples.

\subsection{Implicit measurement experiment}

IAT is used to study the matching between products and images, which mainly uses target samples as stimuli and image words as evaluation words. An implicit measurement experiment was established between the screened sample set and the image semantic set. In the experiment, the subjects will judge whether the image words corresponding to the displayed sample pictures conform to the image words. At the same time, the software will record the judgment data and reaction time data.

\subsection{Analysis and Screening of Implicit Measurement Data}

To reduce the influence of gross error on the data, we analyze the implicit measurement data of each subject and remove the gross error, to get more reliable implicit measurement data. Because the implicit association test is affected by the differences between the subjects, it is necessary to reduce the influence of the differences between the subjects and transform the implicit measurement data into values. Based on the implicit measurement data of each subject, the implicit measurement data of each subject is uniformly converted into $[0,1]$ interval value by formula (1).

$$
F=\frac{f-f_{\text {min }}}{f_{\text {max }}-f_{\text {min }}}
$$

Where $f$ represents the original data, $F$ represents the converted data, $f_{\min }$ represents the minimum value of the data, and $f_{\text {max }}$ represents the maximum value of the data.

\subsection{Establishment of Implicit Data Association}

According to the analysis, a correlation is established between the screened implicit index and the image semantic value. According to the numerical relation of the analysis, the trend relation diagram of each implicit index data under the image semantic value is established. According to the trend relation diagram, the relevant mapping is established to get the change value of the implicit index, to achieve the implicit index after high correlation mapping with the image semantic value.

\subsection{Establishment of Image Extraction Model}

To build a better image extraction model, it is necessary to convert each index of implicit measurement after mapping conversion according to formula (1) again to get the conversion value of each mapping index in $[0,1]$. In the final conversion value, the set of implicit association test indicators $I_{t s}^{\tau}=\left\{I_{t s}^{1}, I_{t s}^{2}, \cdots, I_{t s}^{\tau}\right\}$ (each conversion implicit association test indicator under image vocabulary $\mathrm{S}$ of sample T) and the image value $V_{t s}$ (image value under image vocabulary $\mathrm{S}$ of sample $\mathrm{T}$ ) are used to establish the mapping relationship model, as shown in formula (2).

$$
V_{t s}=i^{1} \cdot I_{t s}^{1}+i^{2} \cdot I_{t s}^{2}+\cdots+i^{\tau} I_{t s}^{\tau}+\theta
$$

Where $\theta$ is a constant term and $i$ is the weight coefficient.

\section{Product Image Mapping Experiment Based on Implicit Measurement Method}

\subsection{Sample and Image Word Screening}

Through to screen sample images, 66 images that can objectively and accurately reflect the sample properties are gained. To reduce the impact of the brand effect on users, the selected sample pictures uniformly remove the logo and others, ensure the angle of each picture taken and the overall part of the picture are consistent and reduce other unrelated to this study. At the same time of collecting sample pictures, image adjectives related to sample pictures are collected. Through the selection and clustering of adjectives, 7 groups of classification results are gained. According to the meaning of the words, seven pairs of adjectives are summed up, which are light - heavy, traditional - front, hard - soft, simple - complex, unique ordinary, stable - lively, firm - fragile.

\subsection{Establishing Image Semantic Space}

The 66 processed UAV sample pictures were investigated by the questionnaire. The questionnaire was designed based on the 5-point Likert scale, and 4 pairs of image words were evaluated. Taking "light-heavy" as an example, -2 represents "very light", -1 represents "some light", 2 represents "very heavy", 1 represents "some heavy" and 0 represents neutral. We will investigate this questionnaire through the Internet, and the questionnaire will prompt you to fill in whether you have bought 
consumer-grade unmanned aerial vehicles, whether you are interested in buying consumer-grade unmanned aerial vehicles, whether you know about consumer-grade unmanned aerial vehicles, and your age and education level. 215 questionnaires were collected. According to situate all the investigated personnel, we carry out classification analysis and statistics. Through the collection, analysis, and exclusion of perceptual evaluation data, the image average value of each group of image vocabulary pairs of unmanned aerial vehicle samples is gained.

\subsection{Screening of Sensitive Samples}

The evaluation values of 4 pairs of image words of 66 samples are arranged according to each, and the image values under each are divided into three categories. Taking "light-heavy" as an example, the image evaluation results can be reflected as "light", "heavy" and "neutral". The samples will be evaluated. The perceptual evaluation at this stage will be expressed by three semantic differences, "light", "heavy" and "neutral". The questionnaire will randomly select 20 students on campus for investigation. Once the data is available, the excluding samples that do not conform to the results of the first stage survey. That is, the perceptual evaluation in the first stage conforms to the "light" sample. If any respondent chooses "neutral" or "heavy" in this survey, it will be excluded to ensure that each sample exactly conforms to the evaluated image, and the error data gained in the first stage of the survey will be excluded. After screening, 5 "light" image evaluation unmanned aerial vehicles, 6 "heavy" image evaluation unmanned aerial vehicles and 4 "neutral" image evaluation unmanned aerial vehicles will be gained. Similarly, the corresponding samples are gained in the same way under other perceptual images.

\subsection{Implicit measurement experiment}

Taking the image of "light-heavy" as an example, the 15 selected samples are subjected to implicit measurement experiments. Each sample corresponds to a word, that is "Light" or "heavy", and two matching states between the samples and the image words are established, that is Coincidence and noncoincidence. Therefore, there will be 30 kinds of experimental in the implicit measurement experiment. In this experiment, 21 industrial design students with a design background were recruited as subjects. Each subject has the knowledge background of Kansei engineering, understands the semantic evaluation of images, and has a good cognitive ability. The age is between 20 and 25 years old.

The experiment was conducted in a closed, quiet, softlit and noiseless laboratory. The specific steps of the experiment are: first, a "+" picture is displayed in the center of the screen for 1 -second to guide the subjects to pay attention. Then a stimulus sample picture appeared for 5 seconds. After the sample picture is presented for 5 seconds, the image words are presented at the bottom of the picture in the picture, and the presentation time is the time selected by the user. Users need to judge the image words, click the left mouse button in line with the sample meaning and click the right mouse button in line with the sample meaning. After completing this, carry out the following experimental stimulation until all sample experiments are completed.

\section{Data processing analysis}

\subsection{Data Analysis of Implicit Association Test}

\subsubsection{Subject Selection Analysis.}

The subjects' image matching and selection under to stimulate each sample target and each image word can well reflect the image attributes of the sample. By counting the selection rate of subjects under each image, as shown in Table 1, it can be seen that when the selection rate of a certain image is high, the selection rate of relative image words is low. When the selection rate of a certain image is close to the median, the selection rate of relative image words is also close to the median. According to the analysis of image matching results, when the sample belongs to the "light" attribute, the light selection rate is higher and the heavy selection rate is lower. When the sample belongs to the "heavy" attribute, the heavy selection rate is higher and the light selection rate is lower. When the sample belongs to the "neutral" attribute, the difference between the light selection rate and the heavy selection rate is small and both are near the median.

Table 1. Image Selection Rate under Each Sample.

\begin{tabular}{cccccccccccccccc}
\hline Sample & 1 & 2 & 3 & 4 & 5 & 6 & 7 & 8 & 9 & 10 & 11 & 12 & 13 & 14 & 15 \\
\hline $\begin{array}{c}\text { Light } \\
\text { selection } \\
\text { Bulky }\end{array}$ & 0.47 & 0.38 & 0.95 & 0.76 & 0.14 & 0.28 & 0.9 & 0.38 & 0.14 & 0.33 & 0 & 0.14 & 1 & 0.95 & 0.14 \\
selection & 0.52 & 0.57 & 0 & 0.19 & 0.81 & 0.61 & 0.09 & 0.66 & 0.81 & 0.66 & 0.9 & 0.85 & 0 & 0.19 & 0.66 \\
\hline
\end{tabular}

\subsubsection{Analysis of reaction time of subjects.}

Based on the theory of implicit association test, it can be known that when the target attribute matches the word, the reaction time of the subjects' selection is shorter, and when the matching is fuzzy, the reaction time of the selection is longer. Based on this theory, when users understand the target sample, the time from to occur perceptual words to the decision-making time is taken as the reaction time, which can fully represent the cognitive decision-making 
time of the subjects. Because of the differences in individual, each subject's reaction time needs to remove its differences before further processing and analysis can be carried out, and reaction time with large errors can be excluded. After getting the reaction time of each subject, gross errors need to be removed. To better judge whether the reaction time data is gross errors, according to the analysis of the data, formula (3) is used to remove, that is the reaction time data greater than 2 times the average value is excluded. After getting the cleaned data, to reduce the influence of the differences among the subjects themselves, formula (1) is used to convert the reaction time of the subjects into interval values [0,1]. After converting the values, averaging the reaction time conversion values of each subject was carried out to get the reaction time conversion data of each sample under to stimulate each image word. By analyzing the data, we can know that when the sample attribute belongs to the clear image of "light" or "heavy", the reaction data is shorter when the matching result is consistent or inconsistent, and when the sample attribute belongs to "neutral", the reaction data is longer when the matching result is consistent or inconsistent.

$$
\mathrm{R}_{\mathrm{i}} \leq 2 \times \frac{\sum_{\mathrm{i}=\mathrm{i}}^{\mathrm{n}} \mathrm{R}_{\mathrm{i}}}{\mathrm{n}}
$$

Where $R$ is the reaction time value.

\subsection{Analysis and Summary}

According to the analysis of the reaction time data of the implicit association test. The value of each data when the sample image attribute is clear is less than the value of each data when the sample image attribute is unclear (that is Neutral). According to the data of all subjects in each trial, the two data of the same sample are added to get the downloaded data of the sample. To introduce implicit data and image values under each sample is analyzed by fitting formula (4), and the fitting trend line between image values and each data is drawn according to the data. From the trend chart, it can be seen that when the image value is in the middle position, the reaction time has a higher value, indicating that when the sample is in the neutral image attribute, the reaction time value is larger than when the sample is in the definite image attribute. When the image value is small, the light selection rate is higher and the heavy selection rate is lower. When the image value is large, the light selection rate is lower and the heavy selection rate is higher. The trend chart is shown in Figure 1 .

$$
Y=C_{0}+C_{1} \cdot X+C_{2} \cdot X^{2}
$$

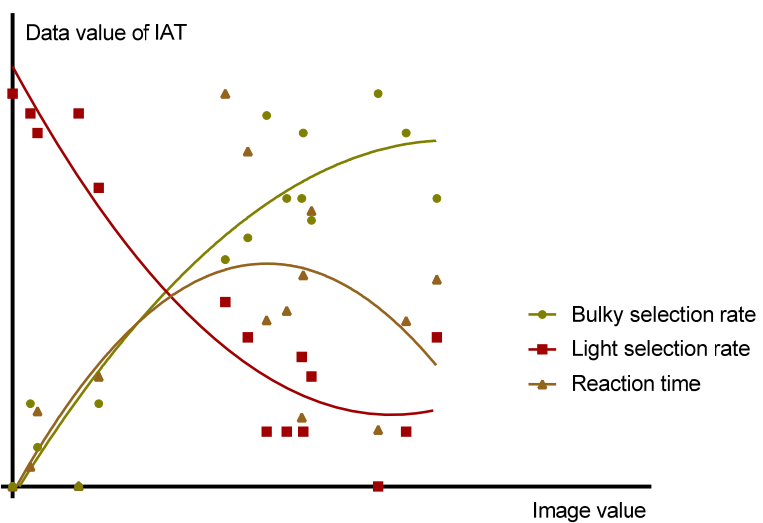

Figure 1. Trend between IAT data and Image value.

\section{Construction of Image Extraction Model Based on Implicit Measurement Data}

According to the analysis of implicit measurement data, it is known the reaction time data and selection data in implicit association tests have a significant correlation with the image attributes of the samples, so the research will select data to establish an image extraction model.

\subsection{Establishment of Correlation of Implicit Measurement Data}

\subsubsection{Establishment of Correlation of Subject Selection Rate Data.}

According to the analysis of the selection rate data, when the image attribute of the sample is clear, to select the sample under different image words is high or low; when the image attribute of the sample is not clear (neutral), to select the sample under different words will be near the median value. According to the analysis results of the selection rate, the high correlation between the selection rate of the subjects and the image is established. The selection rate of the sample under the "light" image vocabulary is defined as a $a_{1}$. And the selection rate under the "heavy" image vocabulary is a $a_{2}$. When $a_{1}>a_{2}$, the image attribute of the sample is "light", the attribute of the sample is "heavy", to establish the formula (5).

$$
A_{i}=\frac{1}{2}-\frac{a_{i 1}-a_{i 2}}{2}
$$

\subsubsection{Establishment of related to Reaction Time.}

Based on the analysis of implicit measurement data, a high correlation model between each data and image value is established. For the analysis of each implicit measurement 
data and image value, the size of each data value range is different. To better establish the relevant model, use formula (1) to convert the implicit measurement data and image value to $[0,1]$ range value. According to the trend between each implicit measurement data and image value reflected in Figure 1, when the image attribute of the sample is not clear, the value of each implicit measurement data is high. According to the results of formula (1), the maximum value is converted to 1 , and the minimum value is converted to 0 . The higher the value is, the closer the conversion value is to 1 , the closer to 0 . Therefore, the formula (6) is established based on this Number, in which the implicit measurement data is defined as $r$, and the selection rate in formula (4) is defined as $a_{1}$ and $a_{2}$. The data after transformation is transformed again to meet the $[0,1]$ interval value, and the t-test analysis is carried out with the image transformation value. The significance of all the transformed implicit measurement data and image value is $p<0.05$, which shows that a high correlation between the implicit measurement data and image, indicating that this formula can establish a high correlation between the selection rate and image value. The conversion data of the implicit association test of each sample is shown in table 2 .

$$
R= \begin{cases}0+\frac{r_{i}}{2} & a_{i 1}>a_{i 2} \\ 1-\frac{r_{i}}{2} & a_{i 1}<a_{i 2}\end{cases}
$$

Table 2. IAT conversion data

\begin{tabular}{cccccccccccccccccc}
\hline Sample & 1 & 2 & 3 & 4 & 5 & 6 & 7 & 8 & 9 & 10 & 11 & 12 & 13 & 14 & 15 & CC & Sig \\
\hline Selection & 0.55 & 0.63 & 0.03 & 0.23 & 0.88 & 0.70 & 0.10 & 0.68 & 0.88 & 0.70 & 1.00 & 0.90 & 0.00 & 0.13 & 0.80 & 0.91 & 0 \\
Reaction & 0.54 & 0.62 & 0.00 & 0.15 & 0.85 & 0.70 & 0.10 & 0.79 & 0.79 & 0.98 & 1.00 & 0.85 & 0.00 & 0.03 & 0.84 & 0.93 & 0 \\
Image & 0.04 & 0.15 & -0.7 & -0.6 & 0.94 & 0.47 & -0.9 & 1.09 & 0.43 & 0.42 & 0.80 & 0.24 & -1.0 & -0.9 & 0.34 & & \\
\hline
\end{tabular}

\subsection{Establishment of Image Extraction Model}

According to the t-test results of each implicit measurement data and image value data, we can know that a significant correlation between the implicit measurement data and image value. Taking the implicit measurement data as the independent variable, the linear between the image semantic value is set up, and the multiple linear equations between the sample perceptual image and each implicit measurement data are gained. According to formula (2) to define image value $V$, the selection rate $I^{1}$, reaction time $I^{2}$, constant $\theta$, each implicit measurement data weight to define selection rate $i^{1}$, reaction time $i^{2}$, then the multiple linear equations is shown in formula (7) below, the sample data is substituted into SPSS for analysis, and the weight value of the gained and implicit measurement data is shown in formula (8).

$$
\begin{gathered}
V=i^{1} \cdot I^{1}+i^{2} \cdot I^{2}+\theta \\
V=0.323 \cdot I^{1}+1.439 \cdot I^{2}-0.914(8)
\end{gathered}
$$

Each implicit measurement data is substituted into Formula (8). The image extraction estimation value of each sample is gained, and the correlation analysis is carried out between the estimation value and the sample image value gained from the questionnaire. The correlation between the estimation value extracted from the implicit association test data and the image value reaches $0.98, p=0.01<0.05$, which shows the effectiveness of the image extraction model. The fitting relation line between the two is shown in Figure 2.

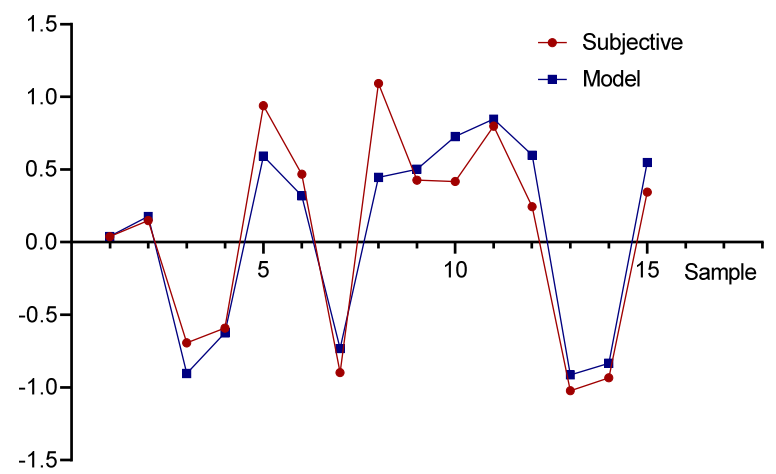

Figure 2. Fitting the between model value and subjective value.

\section{Conclusion}

Based on the implicit association test to analyze the objective data of users' product image cognitive evaluation, the relation between the selection rate and response time and the image attributes of each sample were analyzed. The model between the transformation data of the implicit association test and the image value of the sample is established to verify the validity of the image extraction model. The image acquisition method model based on implicit association test reduces the influence of subjective in user evaluation and provides new thinking and paradigm for the objective decision-making model of the product image. 


\section{Acknowledgments}

Funding: This project was supported by the National Natural Science Foundation of China (grant numbers 51865003) and Guizhou Provincial Science and Technology Plan Project (grant numbers [2018] 5781).

\section{References}

1. Lin, L., Guo, Z.E., \& Yang, M.Q. (2020). Current research situation and trend of product image-based modeling optimization. Packaging Engineering, 41(02), 65-79.

2. Luo, S.J., \& Pan, Y.H. (2007). Review of Theory, Key Technologies and Its Application of Perceptual Image in Product Design. Chinese Journal of Mechanical Engineering, 43(3), 8-13.

3. Jiao, Y.R., \& Qu, Q.X. (2019). A proposal for Kansei knowledge extraction method based on natural language processing technology and online product reviews. Computers in Industry, 108, 1-11.

4. LaPiere, R. T. (1934). Attitudes vs. actions. Social forces, 13(2), 230-237.

5. Yang, M.Q., Lin, L., Chen, Z.A., Wu, L., \& Guo, Z.E. (2020). Research on the construction method of kansei image prediction model based on cognition of EEG and ET. International Journal on Interactive Design and Manufacturing (IJIDeM), 1-21.

6. Nagamachi, M. (1995). Kansei Engineering: A new ergonomic consumer-oriented technology for product development. International Journal of Industrial Ergonomics, 15(1), 3-11.

7. Nagamachi, M. (2002). Kansei engineering as a powerful consumer-oriented technology for product development. Applied Ergonomics, 33(3), 289-294.

8. Yang, C. (2011). A classification-based Kansei engineering system for modeling consumers' affective responses and analyzing product form features. Expert Systems with Applications, 38(9), 11382-11393.

9. Su, J.N., Wang, P., Zhang, S.T., Zhao, A.S., \& Zhou, A.M.(2013). Review of key technologies of product image styling design. Journal of Machine Design, 30(01), 97-100.

10. Greenwald, A. G., McGhee, D. E., \& Schwartz, J. L. (1998). Measuring individual differences in implicit cognition: the implicit association test. Journal of personality and social psychology, 74(6), 1464. 